\title{
Non-eruption of teeth-maxillary hypoplasia-genu valgum syndrome
}

INSERM

\section{Source}

INSERM. (1999). Orphanet: an online rare disease and orphan drug data base. Noneruption of teeth-maxillary hypoplasia-genu valgum syndrome. ORPHA:2972

Noneruption of teeth - maxillary hypoplasia - genu valgum is an extremely rare syndrome that is characterized by multiple unerupted permanent teeth, hypoplasia of the alveolar process and of the maxillo-zygomatic region, severe genu valgum and deformed ears. 